EPJ Web of Conferences 13, 02005 (2011)

DOI: $10.1051 /$ epjconf/20111302005

(c) Owned by the authors, published by EDP Sciences, 2011

\title{
Energy and system-size dependence of the Chiral Magnetic Effect
}

\author{
V.Toneev ${ }^{12, a}$ and V.Voronyuk ${ }^{134}$ \\ 1 Joint Institute for Nuclear Research, 141980 Dubna, Moscow Region, Russia \\ 2 GSI, Helmholtzzentrum für Schwerionenforschung GmbH, 64291 Darmstadt, Germany \\ 3 Bogolyubov Institute for Theoretical Physics, $03680 \mathrm{Kiev}$, Ukraine \\ 4 Institute für Theoretische Physik, Universität Frankfurt, 60438 Germany
}

\begin{abstract}
The energy dependence of the local $\mathcal{P}$ and $C \mathcal{P}$ violation in $\mathrm{Au}+\mathrm{Au}$ and $\mathrm{Cu}+\mathrm{Cu}$ collisions in a large energy range is estimated within a simple phenomenological model. It is expected that at LHC the chiral magnetic effect will be about 20 times weaker than at RHIC. At lower energy range, covered by the low-energy scan at RHIC and future NICA/FAIR facilities, the created magnetic field strength and energy density of deconfined matter are rather high providing necessary conditions for the chiral magnetic effect. However, the particular model for the chiral magnetic effect predicts that this effect should vanish sharply at energy somewhere above the top SPS one. To elucidate CME background effects the Hadron-String-Dynamics (HSD) transport model including electromagnetic fields is put forward. Importance of new planning experiments at LHC and for the low-energy RHIC scan program is emphasized.
\end{abstract}

\section{Introduction}

Until now there is no direct experimental evidence for topological effects in QCD while the existence of nontrivial topological configurations is a fundamental property of the gauge theory. Transitions between different topological states occur with the local violation of the $\mathcal{P}$ and $C \mathcal{P}$ symmetry. Due to chiral anomaly the interplay of these topological configurations with (chiral) quarks results in asymmetry between left- and right-handed quarks. Such chiral asymmetry coupled to a strong magnetic field, created by colliding nuclei perpendicularly to the reaction plane, induces a current of electric charge along the direction of a magnetic field thereby separating particles of opposite charges with respect to the reaction plane. Thus, as was argued in Refs. [1-5] the topological effects in QCD may be observed in heavy ion collisions directly in the presence of very intense external electromagnetic fields due to the "Chiral Magnetic Effect" (CME) as a manifestation of spontaneous violation of the $C \mathcal{P}$ symmetry. First experimental evidence for the CME identified via the observed charge separation effect with respect to the reaction plane was presented by the STAR Collaboration at RHIC [6]. In this paper we analyze the STAR data in a simple phenomenological way to estimate a possibility observing the CME in the larger energy range, from the LHC to FAIR/NICA energies. We also make a step toward a dynamical estimate of the CME background based on the nonequalibrium Hadron-String-Dynamics (HSD) microscopical transport approach [7] including electro-magnetic field.

\footnotetext{
a e-mail: toneev@theor.jinr.ru
}

\section{Phenomenological estimates of the CME}

\subsection{Model assumptions}

Qualitatively the CME may be estimated as follows.

We consider the saturation momentum $Q_{s}$ as a characteristic scale of the process [1], so the transverse momentum of particles $p_{t} \sim Q_{s}$. Then the total transverse energy per unit rapidity at mid-rapidity deposited at the formation of hot matter is expressed through the overlapping surface of two colliding nuclei in the transverse plane $S$

$$
\frac{d E_{T}}{d y} \sim \epsilon \cdot V=\epsilon \cdot \Delta z \cdot S=Q_{s} \cdot\left(Q_{s}^{2} S\right) .
$$

Here the energy density and longitudinal size $\Delta z \simeq \Delta \tau \simeq$ $1 / Q_{s}$ are taken in order of magnitude as follows $\epsilon \sim Q_{s}^{4}$ and $\Delta z \sim 1 / Q_{s}$. The value in brackets is proportional to the hadron multiplicity $\left(Q_{s}^{2} S\right) \sim d N_{\text {hadrons }} / d y$, thus we get

$$
\frac{d E_{T}}{d y} \sim Q_{s} \cdot \frac{d N_{\text {hadrons }}}{d y},
$$

which simply tells us that the total transverse energy per unit rapidity is equal to the total number of produced hadrons per unit rapidity times their average transverse momentum.

The topological charge (winding number) generated in random motion during the time $\tau_{B}$ when the magnetic field is present may be estimated as

$$
n_{w} \equiv \sqrt{Q^{2}}=\sqrt{\Gamma_{S} \cdot V \cdot \tau_{B}}
$$

where $\Gamma_{S}$ is the sphaleron transition rate. Sphalerons describe a one dimensional random walk in the topological 
number space. At weak coupling, $\Gamma_{S} \sim \alpha_{s}^{5} T^{4}$. Little is known about the rate of topological charge diffusion at strong coupling $\lambda=g^{2} N_{c}$; however in SUSY Yang-Mills theory the diffusion rate of topological charge can be evaluated explicitly and is given by $\Gamma_{S} \sim \lambda^{2} T^{4}$.

The initial temperature $T_{0}$ of the produced matter at time $\tau \simeq 1 / Q_{s}$ is proportional to the saturation momentum $Q_{s}, T_{0}=c Q_{s}$. Neglecting the expansion time and the corresponding time dependence of the temperature we can roughly get

$$
n_{w} \equiv \sqrt{Q^{2}} \sim \sqrt{\frac{d N_{\text {hadrons }}}{d y}} \cdot \sqrt{Q_{s} \tau_{B}} .
$$

Since sizable sphaleron transitions occur only in the deconfined phase, the time $\tau_{B}$ in Eq. (4) is really the smallest lifetime between the strong magnetic field $\tilde{\tau}_{B}$ one and the lifetime of deconfined matter $\tau_{\epsilon}$ :

$$
\tau_{B}=\min \left\{\tilde{\tau}_{B}, \tau_{\epsilon}\right\}
$$

The measured charged particle asymmetry is associated with the averaged correlator $a$ by the following relation [8]:

$$
\begin{aligned}
& \left\langle\cos \left(\psi_{\alpha}+\psi_{\beta}-2 \Psi_{R P}\right)\right\rangle= \\
= & \left\langle\cos \left(\psi_{\alpha}+\psi_{\beta}-2 \psi_{c}\right)\right\rangle / v_{2, c}=v_{1, \alpha} v_{1, \beta}-a_{\alpha} a_{\beta},
\end{aligned}
$$

where $\Psi_{R P}$ is the azimuthal angle of the reaction plane defined by the beam axis and the line joining the centers of colliding nuclei and averaging in (6) is carried out over the whole event ensemble. The second equality in (6) corresponds to azimuthal measurements with respect to particle of type $c$ extracted from three-body correlation analysis [8], $v_{1}$ and $v_{2}$ are the directed and elliptic flow parameters, respectively. According to Ref. [1] an average correlator $a=\sqrt{a_{\alpha} a_{\beta}}$ is related to the topological charge, $n_{w}$, as

$$
a \sim \frac{n_{w}}{d N_{\text {hadrons }} / d y} .
$$

This estimate neglects absorption and rescattering in dense matter responsible for the difference of magnitudes between the same and opposite charge correlations and so is very rough. Nevertheless, for the purposes of our estimate, the use of Eqs. (4) and (7) yields the relation

$$
a \sim \frac{\sqrt{Q_{s} \tau_{B}}}{\sqrt{d N_{\text {hadrons }} / d y}},
$$

on which our consideration is based.

\subsection{Energy dependence of the CME}

Let us now come to numerical estimates. Using the energy dependence $Q_{s}^{2} \sim s_{N N}^{1 / 8} \sim d N_{\text {hadrons }} / d y$ deduced from RHIC and HERA $[9,10]$ data we finally get for the energy dependence

$$
a \sim \sqrt{\frac{\tau_{B}}{Q_{s}}} \sim\left(\sqrt{s_{N N}}\right)^{-1 / 16} \cdot \sqrt{\tau_{B}} .
$$

Thus, the direct energy dependence is comparatively weak. Results of dynamical heavy-ion calculations of the magnetic field at the central point of the transverse overlapping region of colliding nuclei and energy density of created particles are presented in Figs. 1 and 2, respectively. Here for a field estimate we follow Ref. [11] basing on the UrQMD model $[12,13]$ and applying the electromagnetic Lienard-Wiechert potential with the retardation condition for the magnetic field. As is seen, at the impact parameter $b=10 \mathrm{fm}$ the maximal strength of the dominant magnetic field component $B_{y}$ (being perpendicular to the reaction plane) is decreased in $\mathrm{Au}+\mathrm{Au}$ collisions by the factor of about 10 , when one proceeds from $\sqrt{s_{N N}}=200 \mathrm{GeV}$ to $E_{l a b}=11 \mathrm{GeV}$, while for the created particle energy density $\varepsilon$ in the central box this factor is 250 i.e. noticeably higher.
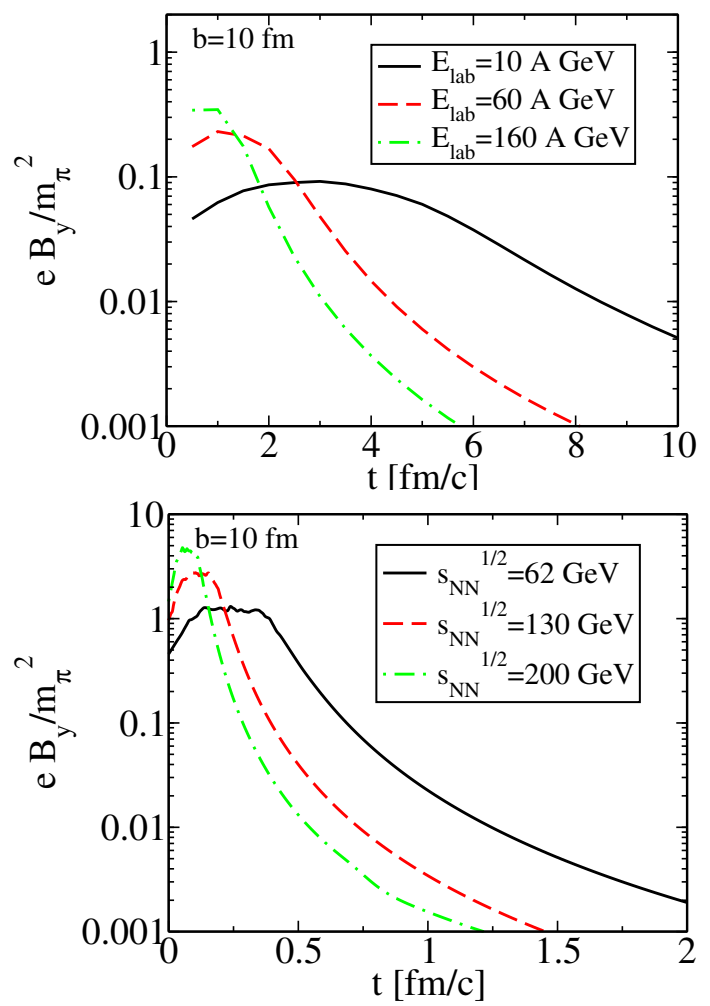

Fig. 1. The time evolution of the magnetic field strength $e B_{y}$ at the central region in $\mathrm{Au}+\mathrm{Au}$ collisions with the impact parameter $b=$ $10 \mathrm{fm}$ for different bombarding energies. Calculations are carried out within the UrQMD model [12,13] (for a detail see [11]).

To use Eq. (8) we need to identify the impact parameter, saturation momentum and multiplicity at a specific centrality. These can be found in Ref. [10] where the Glauber calculations were done. As a reference point we choose $b=10 \mathrm{fm}$ in our subsequent consideration.

The measured value of $\left\langle\cos \left(\psi_{\alpha}+\psi_{\beta}-2 \Psi_{R P}\right)\right\rangle$ for the same charge particles from $\mathrm{Au}+\mathrm{Au}\left(\sqrt{s_{N N}}=200 \mathrm{GeV}\right)$ collisions at the impact parameter $b=10 \mathrm{fm}(40-50 \%$ centrality interval $)$ is $-(0.312 \pm 0.027) \cdot 10^{-3}[6]$. Appropriate number for $\sqrt{s_{N N}}=62 \mathrm{GeV}$ seems to be a little bit larger 

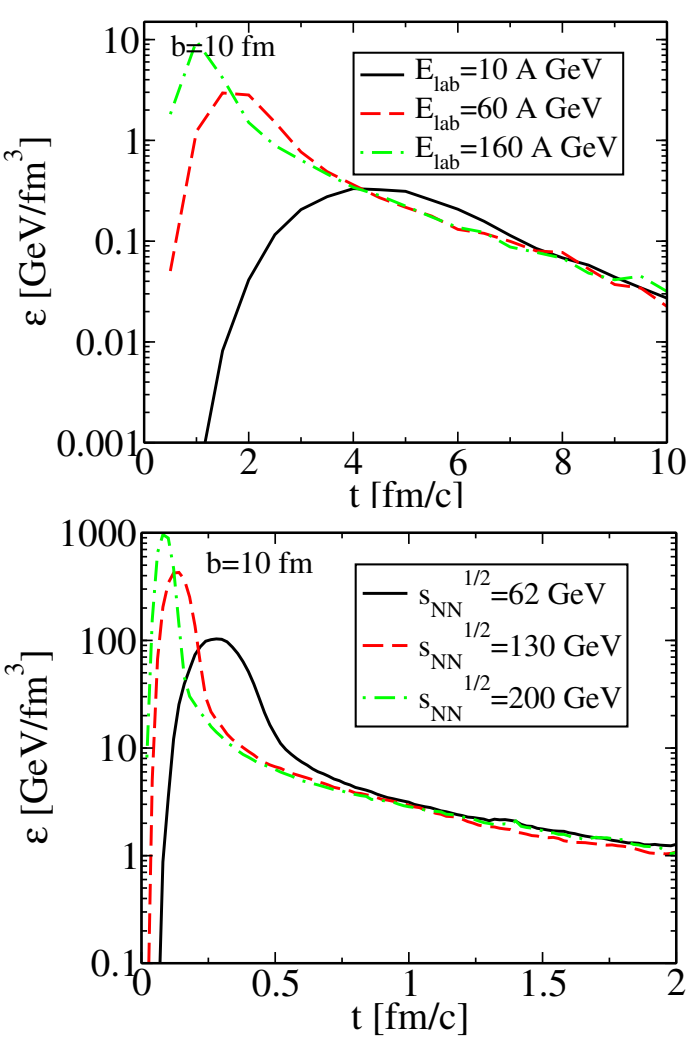

Fig. 2. The time evolution of the energy density $\varepsilon$ of created particles in the Lorentz-contracted box with the $2 \mathrm{fm}$ side at the central point of overlapping region. The impact parameter $b=10 \mathrm{fm}$.

but for $\mathrm{Cu}+\mathrm{Cu}$ collisions the effect is definitely stronger [6]. Thus, ignoring any final state interactions with medium, assuming $a_{\alpha}=a_{\beta}=a$ and neglecting the directed flow $v_{1 a}=v_{1 b}=0$ we get from Eq. (6) $a_{\text {exp }}^{2}=0.31 \cdot 10^{-3}$ for the maximal RHIC energy. Using numbers for the $\sqrt{s_{N N}}=200$ $\mathrm{GeV}$ reference case, from Eq. (9) we may quantify the $C \mathcal{P}$ violation effect by the correlator

$$
a^{2}=K_{A u}\left(\sqrt{s_{N N}}\right)^{-1 / 8} \cdot \tau_{B} .
$$

The normalization constant $K_{A u}$ can be tunned at the reference energy $\sqrt{s_{N N}}=200 \mathrm{GeV}$ from the inverse relation and experimental value $a_{\text {exp }}$ at this energy for $b=10 \mathrm{fm}$

$$
K_{A u}=\frac{a_{\text {exp }}^{2} \cdot(200)^{1 / 8}}{\tau_{B}(200)} .
$$

The lifetime $\tau_{B}$ may be defined as the time during which the magnetic field is above the critical value needed to support a fermion Landau level on the domain wall $e B_{\text {crit }}=$ $2 \pi / S_{d}$, where $S_{d}$ is the domain wall area. Since the size of the domain wall is not reliably known, it is hard to pin down the number, but it should be of the order of $m_{\pi}^{2}$. Honestly, we have to treat it as a free parameter.

Indeed the size of the topological defect (say e.g., a sphaleron) in the region between $T_{c}$ and $2 T_{c}$ is very uncertain. At weak coupling, the size is determined by the magnetic screening mass and it is $\sim 1 /\left(\alpha_{s} T\right)$. If one plugs
Table 1. Estimated parameters for the $C \mathcal{P}$ violation effect in $\mathrm{Au}+\mathrm{Au}$ collisions at centrality (40-50)\% with the critical field $e B_{\text {crit }}=0.2 m_{\pi}^{2}$.

\begin{tabular}{|c|c|c|c|c|}
\hline$\sqrt{s_{N N}} \mathrm{GeV}$ & $s_{N N}^{1 / 16}$ & $\tilde{\tau}_{B}, \mathrm{fm} / \mathrm{c}$ & $\tau_{\epsilon}, \mathrm{fm} / \mathrm{c}$ & $a^{2}$ \\
\hline $4.5 \cdot 10^{3}$ & 2.86 & 0.018 & $>1$ & $0.016 \cdot 10^{-4}$ \\
200 & 1.94 & 0.24 & $>2$ & $0.31 \cdot 10^{-3}$ \\
130 & 1.84 & 0.33 & $\sim 2.3$ & $0.45 \cdot 10^{-3}$ \\
62 & 1.68 & 0.62 & $\sim 2.2$ & $0.93 \cdot 10^{-3}$ \\
17.9 & 1.43 & 1.41 & $\sim 2$. & $2.48 \cdot 10^{-3}$ \\
11. & 1.35 & 1.66 & $\sim 1.9$ & $3.10 \cdot 10^{-3}$ \\
4.7 & 1.21 & 0. & 0. & 0. \\
\hline
\end{tabular}

$\alpha_{s} \approx 0.5$ and $T=200 \mathrm{MeV}$, the size is of about $2 \mathrm{fm}$ and then the threshold field is very small $e B_{y} \sim\left(\alpha_{s} T\right)^{2} \sim$ $0.2 m_{\pi}^{2}$.

On the other hand, we know that between $T_{c}$ and $2 T_{c}$ the magnetic screening mass which determines the size of the sphaleron is not small as expected from the perturbative theory, $\alpha_{s} T$, but from the lattice it is numerically large till about $5 T_{c}$. This would increase the threshold to $20 \mathrm{~m}_{\pi}^{2}$, however the relation between magnetic mass and the sphaleron size is valid only as long as the coupling is weak.

All we can say it is perhaps in between $(0.2-20) \mathrm{m}_{\pi}^{2}$. Eventually lattice QCD calculations may clear this up.

The upper bound on the magnetic strength $e B_{\text {crit }}=$ $20 m_{\pi}^{2}$ results in $\tau_{B}=0$ even for the RHIC energy and therefore in this case the CME should not be observable at all in this energy range. The time evolution of the magnetic field and energy density, $\varepsilon$, of newly created hadrons are presented in Figs. 1 and 2. The extracted values of $\tau_{B}$ defined by the constraints $e B_{y}>0.2 m_{\pi}^{2}$ and $\tau_{\epsilon}(\epsilon>$ $1 \mathrm{GeV} / \mathrm{fm}^{3}$ ) are summed in Tabl. 1 . For the reference energy and the minimal magnetic field constraint we have

$$
K_{A u}=2.52 \cdot 10^{-3} \text {. }
$$

If lifetimes are known for all energies one can estimate the $C \mathcal{P}$ violation effect through the $a^{2}$ excitation function.

¿From the first glimpse as follows from Tabl. 1, in the case of $e B_{c r i t}=0.2 m_{\pi}^{2}$ the interaction time $\tau_{B}$ is defined solely by evolution of the magnetic field since $\tilde{\tau}_{B}<\tau_{\varepsilon}$ whereas $\tau_{\varepsilon} \approx 2 \mathrm{fm}$ independent of $\sqrt{s_{N N}}$. The expected $\mathrm{CME}$ for $\mathrm{Au}+\mathrm{Au}$ at $b=10 \mathrm{fm}$ (see the last column in Tabl. 1) monotonously increases when $\sqrt{s_{N N}}$ goes down but then sharply vanishes exhibiting a shallow maximum in the range between near the top SPS and NICA energies. The position of CME maximum and its magnitude depend on the cut level which just defines $\tilde{\tau}_{B}$. The decrease of the $e B_{y}$ bound to $0.02 m_{\pi}^{2}$ shifts the maximum toward lower energy $\sqrt{s_{N N}}$ and enhances its magnitude. In an opposite limit when results are extrapolated to the LHC energy, the CME falls down by a factor of about 20 with respect to the RHIC energy. This result is quite understandable. The $\mathrm{CME}$ is mainly defined by the relaxation time of the magnetic field which is concentrated in the Lorentz contracted nuclear region $\sim 2 R / \gamma$. Therefore, the CME inversely proportional to the colliding energy, $\sim 1 / \sqrt{s_{N N}}$ and proceed- 
ing from the RHIC to LHC energy we roughly get the suppression factor about 4.5/0.2 22 .

There is one worrying point here. Proceeding from $\sqrt{s_{N N}}=200$ to $62 \mathrm{GeV}$ the predicted value of $a^{2}$ for $b=10$ $\mathrm{fm}$ increases in three times though not more $20 \%$ growth has been observed in these collisions in the recent experiment [6]. This essential disagreement cannot be removed by a simple variation of $e B_{\text {crit }}$. One may try to explain this correlator overestimation at $\sqrt{s_{N N}}=62 \mathrm{GeV}$ by an irrelevant choice of the energy dependence of multiplicity in Eq. (10). For the correlator ratio at these two energies we have

$$
\begin{aligned}
\frac{a^{2}(200)}{a^{2}(62)} & =\frac{\tau_{B}(200)}{\tau_{B}(62)}\left(\frac{62}{200}\right)^{1 / 8} \\
& =0.387(0.31)^{\beta} \approx 0.72 .
\end{aligned}
$$

where we use lifetime values from Tabl. 1 and experimental values for correlators [6], $\beta \equiv 1 / 8$. As follows from Eq. (13), to explain the experiment the exponent should be negative, $\beta<0$. Therefore, the fast growth of $\tau_{B}$ with the energy decrease cannot be compensated by uncertainty in energy dependence of the correlator $a$.

Uncertainty in the choice of the impact parameter does not help us to solve this issue as well. As is seen in Fig. 3 the maximal strength of the magnetic field slightly increases with the impact parameter but the lifetime of strong magnetic field $\tilde{\tau}_{B}$ practically is independent of the impact parameter (for example, look at the level of $e B_{\text {crit }}=0.2 \mathrm{~m}_{\pi}^{2}$ ). It will be used below as an estimate of the $b$-dependence of the CME which is roughly proportional to $b$ [3].

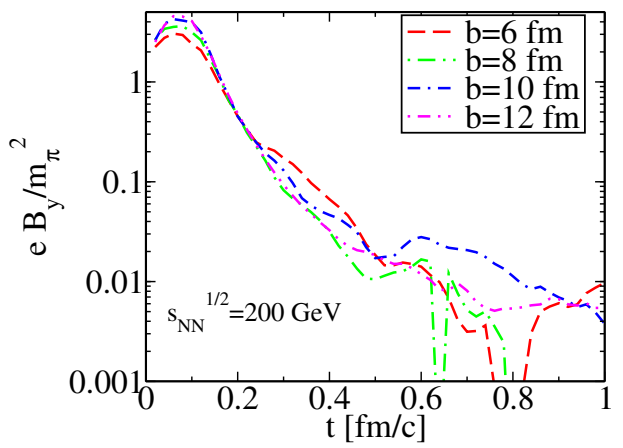

Fig. 3. The time evolution of the magnetic field strength $e B_{y}$ at the central region in $\mathrm{Au}-\mathrm{Au}(200 \mathrm{GeV})$ collisions for different impact parameters.

As follows from Eq. (13), to eliminate model discrepancy between two RHIC energies the lifetimes should be comparatively close to each other, $\tau_{B}(62) \approx 1.2 \tau_{B}(200)$. One may try to fit this ratio by the variation of $e B_{\text {crit }}$. As it is seen from Table 1 at $e B_{\text {crit }}=0.2 \mathrm{~m}_{\pi}^{2}$ we have the $\tau_{B}(62) / \tau_{B}(200) \approx 3.0$. The needed time ratio, 1.2 , is achieved at $e B_{\text {crit }} \sim 1.05 m_{\pi}^{2}$, very close to the maximal magnetic field of $\sim 1.2 \mathrm{~m}_{\pi}^{2}$ for $\sqrt{s_{N N}}=62 \mathrm{GeV}$ (see Fig.1). It is evident that if the low-bound field $e B_{\text {crit }}$ coincides with the maximal one the CME should not be observable. For more accurate tuning new experimental data at $\sqrt{s_{N N}} \lesssim 62$
$\mathrm{GeV}$ are very needed. The decrease of the critical field till $e B_{\text {crit }} \sim 0.01 m_{\pi}^{2}$ makes $\tilde{\tau}_{B}$ larger; however, the condition $\tilde{\tau}_{B}<\tau_{\varepsilon}$ which could improved situation for $\tau_{B}$ is not yet achieved at $\sqrt{s_{N N}}=62 \mathrm{GeV}$. Unfortunately, the influence of this lower bound of the $e B_{y}$ insufficient for manifestation of this effect.

CME assumes that there are soft equilibrated quarksgluons. But it is hard to think that there is a quark-gluon equilibration in the very early state and the concept of the initial time is introduced [3]. It is used to suppose that an equilibrium state is achieved at the expansion stage which starts just after passing the maximum in the energy density evolution. Thus, the $\tau_{\varepsilon}$ value should be count off from this time moment $t_{i}\left(\sqrt{s_{N N}}\right)$ rather than from zero. This correction makes shorter the lifetime by $t_{i, \varepsilon}=0.08$ and $\sim 0.32$ fm for $\sqrt{s_{N N}}=200$ and $62 \mathrm{GeV}$, respectively, as it follows from Fig. 2. Thus, the lifetime ratio at two energies discussed is getting less as the ratios, $\tau_{B}(62) / \tau_{B}(200) \approx$ $(0.62-0.32) /(0.24-0.08) \approx 2$ instead of the mentioned $\approx 3$.0. This softens the dependence of $a^{2}(200) / a^{2}(62)$ on $\sqrt{s_{N N}}$ but it still is not sufficient.

According to Ref. [3] the initial time is estimated as $t_{i} \sim 1 / Q_{s}$, where $Q_{s} \sim s_{N N}^{1 / 16}$, as noted above. Normalizing the initial time at $\sqrt{s_{N N}}=200 \mathrm{GeV}$ to $t_{i, \varepsilon}=0.08 \mathrm{fm}$ we get $\tau_{B}=0.16 \mathrm{fm}$ for $62 \mathrm{GeV}$. This shift is too short to influence essentially on the $\tau_{B}(200) / \tau_{B}(62)$ ratio. With reference to the Lorentz contraction, other scenario maybe proposed as $t_{i} \sim 1 / \sqrt{s_{N N}}[3,14]$. Under similar normalization we arrive at $t_{i} \approx 0.27 \mathrm{fm}$ which is rather close to $t_{i, \varepsilon}$ at $62 \mathrm{GeV}$ in the above estimate and therefore also is unsatisfactory.

Now let us try to combine two last scenarios by changing the low bound of the magnetic field $e B_{\text {crit }}$ and simultaneously shifting the appropriate interaction time to the initial moment defined by the energy density of created quarks, $\tau_{B}=\tilde{\tau}_{B}\left(e B_{c r i t}\right)-t_{i, \varepsilon}$. It is turned out that the equality (13) is satisfied if $e B_{\text {crit }} \approx 0.7 \mathrm{~m}_{\pi}^{2}$. Using the value of $\tau_{B}(200)$ obtained in this analysis one can recalculate the coefficient in Eq. (10), $K=6.05 \cdot 10^{-3}$, and therefore find the correlator $a$ at any energy. In principle, similar analysis may be repeated for other impact parameters to consider the $b$-dependence of the CME. As was shown in Refs. [3, $14]$ the CME roughly is linear in $b / R$. Taking this as a hypothesis we evaluate the centrality dependence of the CME fitting this line to points $b=10 \mathrm{fm}$ (or centrality $(40-50) \%$ ) to be estimated in our model and $b=0$ where the CME is zero. The results are presented in Fig. 4 for $\mathrm{Au}+\mathrm{Au}$ collisions at three energies.

As it is seen the calculated lines quite reasonably reproduce the measured points of azimuthal asymmetry of charge separation for $\mathrm{Au}+\mathrm{Au}$ collisions at $\sqrt{s_{N N}}=200$ and $62 \mathrm{GeV}$. The chosen value of $e B_{\text {crit }}=0.7 \mathrm{~m}_{\pi}^{2}$ results in absence of the CME at the top SPS energy because the critical magnetic field practically coincides with the maximal field at this bombarding energy (see Fig. 1). The CME at the LHC energy is expected to be less by a factor of about 20 as compared to that at the RHIC energy. Note that at the LHC energy we applied a simplified semi-analytical model [11] for magnetic field creation and assumed $t_{i, \varepsilon}=0$. Thus, 


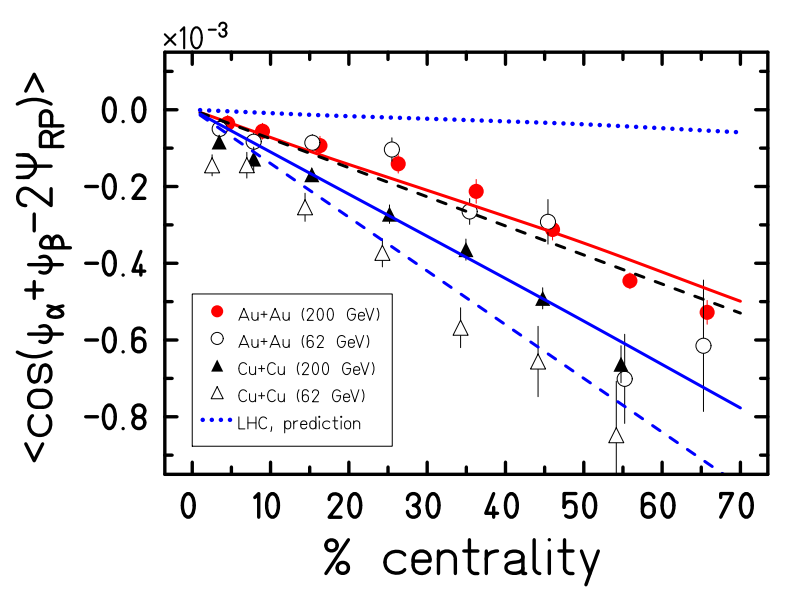

Fig. 4. Centrality dependence of the CME. Experimental points for $\mathrm{Au}+\mathrm{Au}$ and $\mathrm{Cu}+\mathrm{Cu}$ collisions are from [6]. The dotted line is our prediction for $\mathrm{Au}+\mathrm{Au}$ collisions at the LHC energy.

we consider this LHC estimate as an upper limit for the CME.

\subsection{System-size dependence of the CME}

Similar analysis may be repeated for $\mathrm{Cu}+\mathrm{Cu}$ collisions basing on available RHIC measurements at two collision energies. Here one remark is in order. An enhancement of the $\mathrm{CME}$ in $\mathrm{Cu}+\mathrm{Cu}$ collisions with respect to $\mathrm{Au}+\mathrm{Au}$ ones was seen experimentally at the same centrality [6] but not at the same impact parameter. As follows from the Glauber calculations [15], the impact parameter $b=10$ fm for gold reactions corresponds to centrality (40-50)\% while the same centrality for copper collisions matches $b=4.2 \mathrm{fm}$. As is seen in Fig. 5 the time distributions of the magnetic field and energy density for $\mathrm{Cu}+\mathrm{Cu}$ collisions look very similar to that for $\mathrm{Au}+\mathrm{Au}$ ones but lifetimes, both $\tilde{\tau}_{B}$ and $\tau_{\varepsilon}$, are shorter in the $\mathrm{Cu}+\mathrm{Cu}$ case. The extracted lifetimes and other characteristics for $e B_{c r i t}=0.2 m_{\pi}^{2}\left(K_{C u}=6.34 \cdot 10^{-3}\right)$ are presented in Tabl. 2. We meet again the same problem: one should compensate a too strong energy dependence of the model correlators by the proper definition of lifetimes. Defining the lifetime in the same manner as for $\mathrm{Au}+\mathrm{Au}$ collisions $\tau_{B}=\tilde{\tau}_{B}\left(e B_{c r i t}\right)-t_{i, \varepsilon}$ the lifetime ratio at $e B_{\text {crit }}=0.3 m_{\pi}^{2}$ for $\mathrm{Cu}+\mathrm{Cu}$ collisions $\tau_{B}(62) / \tau_{B}(200)=$ $(0.35-0.21) /(0.14-0.06)$ is turned out to be very close to experimental one. In this case $K_{C u}=11.9 \cdot 10^{-3}$. In the linear approximation with the reference point at $b=4.2 \mathrm{fm}$, one may draw the centrality dependence of the CME for $\mathrm{Cu}+\mathrm{Cu}$ collisions shown also in Fig. 4 which is in a reasonable agreement with the experiment. Note that $e B_{\text {crit }}=0.3$ $m_{\pi}^{2}$ which is slightly above the maximal magnetic field at $\sqrt{s_{N N}}=62 \mathrm{GeV}$ implies that the $\mathrm{CME}$ for $\mathrm{Cu}+\mathrm{Cu}$ collisions will not be observable even at the top SPS energy.

From dimensionality arguments the system-size dependence of the chiral magnetic effect (at the same all other conditions) would be expected to be defined by the surface $S \equiv S_{\mathrm{A}}(b)$ of an "almond"-like transverse area of overlapping nuclei since both the high magnetic field and de-
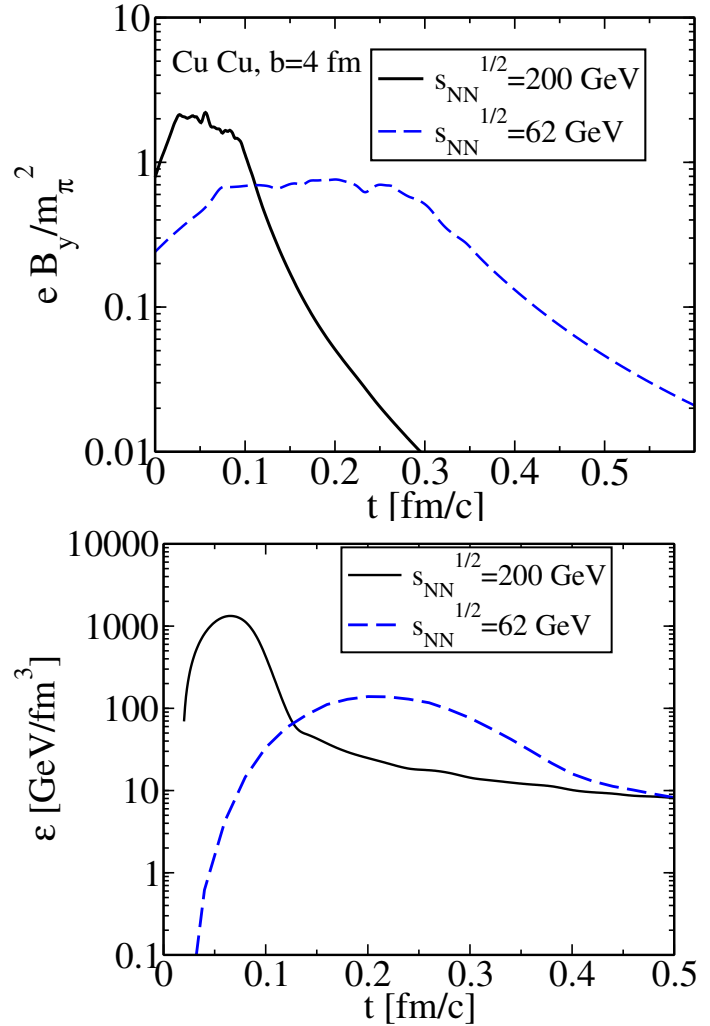

Fig. 5. The time evolution of the magnetic field strength $e B_{y}$ and deconfined energy density $\varepsilon$ at the central region in $\mathrm{Cu}+\mathrm{Cu}$ collisions at $\sqrt{s_{N N}}=200$ and $62 \mathrm{GeV}$ for centrality $(40-50) \%(b=$ $4.2 \mathrm{fm}$ ).

Table 2. Estimated parameters for the $C \mathcal{P}$ violation effect for $\mathrm{Cu}+\mathrm{Cu}(\mathrm{b}=4.2 \mathrm{fm})$ collisions with $e B_{\text {crit }}=0.2 \mathrm{~m}_{\pi}^{2}$.

\begin{tabular}{|c|c|c|c|c|}
\hline$\sqrt{s_{N N}} \mathrm{GeV}$ & $s_{N N}^{1 / 16}$ & $\tau_{B}, \mathrm{fm} / \mathrm{c}$ & $\tau_{\epsilon}, \mathrm{fm} / \mathrm{c}$ & $a^{2}$ \\
\hline 200 & 1.94 & 0.15 & $>2$. & $0.49 \cdot 10^{-3}$ \\
62 & 1.68 & 0.37 & $>2$. & $1.40 \cdot 10^{-3}$ \\
\hline
\end{tabular}

confined matter are needed for this effect. The magnetic field was evaluated in the the center of the overlapping region but as was shown in Ref. [11] the studied $e B_{y}$ component is quite homogeneous along $y$ of this "almond". Above, say in the $\sqrt{S_{N N}}$-analysis of $\mathrm{Au}+\mathrm{Au}$ collisions, the area $S_{\mathrm{Au}}(\mathrm{b}=10 \mathrm{fm})$ does not depend on the energy and it is involved into the coefficient $K$, Eq. (11). Using for "almond" area a rough estimate as for sharp boundary overlapping discs of radius $R=r_{0} A^{1 / 3}$, namely $S \equiv S_{A}(b)=$ $\pi \sqrt{R^{2}-(b / 2)^{2}}(R-b / 2)$, we have $S_{\mathrm{Cu}}(b=4.2) / S_{\mathrm{Au}}(b=$ $10) \approx 1.65$ which seems to be consistent with experimental ratio of the CME at these two points. Thus, if one neglects the lifetime on the system-size and keep only the renormalized coefficient $K_{\mathrm{Cu}}=K_{\mathrm{Au}} S_{\mathrm{Cu}}(b=4.2) / S_{\mathrm{Au}}(b=$ $10)=1.65 K_{\mathrm{Au}}$ for the Au curve at $\sqrt{s_{N N}}=200 \mathrm{GeV}$ in Fig.4 we are able to reproduce rather well the results for $\mathrm{Cu}+\mathrm{Cu}(200 \mathrm{GeV})$. Note that this $\mathrm{Au}$ curve was obtained for $e B_{c r i t}=0.7 m_{\pi}^{2}$ and non-zero initial time $t_{i, \varepsilon}$. Thus, con- 
ditions of the compared events are not identical and this success cannot be repeated for $\mathrm{Cu}+\mathrm{Cu}(62 \mathrm{GeV})$ collisions where maximum of the magnetic field is lower than $0.7 \mathrm{~m}_{\pi}^{2}$. The above rough estimate essentially depends on the $r_{0}$ parameter. From our analysis of the centrality dependence of the CME at $\sqrt{s_{N N}}=200 \mathrm{GeV}$ at $e B_{\text {crit }}=0.2 \mathrm{~m}_{\pi}^{2}$ we have $K_{\mathrm{Cu}}(b=4.2) / K_{\mathrm{Au}}(b=10)=6.34 \cdot 10^{-3} / 2.52 \cdot 10^{-3} \approx 2.5$ which strongly differs from the above estimate. Therefore, the $\mathrm{Cu}$ enhancement effect is not only a geometric one. In this case if the reference point in centrality is considered, the energy dependence should be taking into account. One should note that in a general case the saturation momentum $Q_{s}^{2}$ is weakly $A$-dependent as well what was neglected in our consideration.

\section{Towards a kinetic approach to the CME background}

The discussed CME signal, the electric charge asymmetry with respect to the reaction plane, may originate not only from the spontaneous local $\mathrm{CP}$ violation but also be simulated by other possible effects. In this respect it is important to consider the CME background. We shall do that considering a full evolution of nucleus-nucleus collisions in terms of the HSD transport model [7] but including formation of electromagnetic field as well as its evolution and impact on particle propagation.

Generalized on-shell transport equations for strongly interacting particles in the presence of the electromagnetic fields can be written as

$$
\begin{aligned}
\left\{\frac{\partial}{\partial t}\right. & \left.+\left(\nabla_{\mathbf{p}} \mathbf{U}\right) \nabla_{\mathbf{r}}-\left(\nabla_{\mathbf{r}} \mathbf{U}+q \mathbf{v} \times(\nabla \times \mathbf{A})\right) \nabla_{\mathbf{p}}\right\} f(\mathbf{r}, \mathbf{p}, t) \\
& =I_{\text {coll }}\left(f, f_{1}, \ldots f_{N}\right)
\end{aligned}
$$

which are supplemented by equations for magnetic and electric fields

$$
\mathbf{B}=\nabla \times \mathbf{A}, \quad \mathbf{E}=-\nabla \Phi-\frac{\partial \mathbf{A}}{\partial t} .
$$

The hadronic mean-field $U \sim \operatorname{Re}\left(\Sigma^{r e t}\right) / 2 p_{0}$ here has an additional term from the 4-vector electromagnetic potential $A=\{\Phi, \mathbf{A}\}$. The general solution of the wave equations (15) with charge distribution $\rho(\mathbf{r}, t)=q n$ and current $\mathbf{j}(\mathbf{r}, t)=q \mathbf{v}$ are

$$
\Phi(\mathbf{r}, t)=\frac{1}{4 \pi} \int \frac{\rho\left(\mathbf{r}^{\prime}, t^{\prime}\right) \delta\left(t-t^{\prime}-\left|\mathbf{r}-\mathbf{r}^{\prime}\right| / c\right)}{\left|\mathbf{r}-\mathbf{r}^{\prime}\right|} d^{3} r^{\prime} d t^{\prime}(16)
$$

for the electromagnetic potential $\Phi(\mathbf{r}, t)$ and

$$
\mathbf{A}(\mathbf{r}, t)=\frac{1}{4 \pi} \int \frac{\mathbf{j}\left(\mathbf{r}^{\prime}, t^{\prime}\right) \delta\left(t-t^{\prime}-\left|\mathbf{r}-\mathbf{r}^{\prime}\right| / c\right)}{\left|\mathbf{r}-\mathbf{r}^{\prime}\right|} d^{3} r^{\prime} d t^{\prime}(17)
$$

for the vector potential. For moving point-like charges, one gets

$$
\rho(\mathbf{r}, t)=q \delta(\mathbf{r}-\mathbf{r}(t)) ; \quad \mathbf{j}(\mathbf{r}, t)=q \mathbf{v}(t) \delta(\mathbf{r}-\mathbf{r}(t))
$$
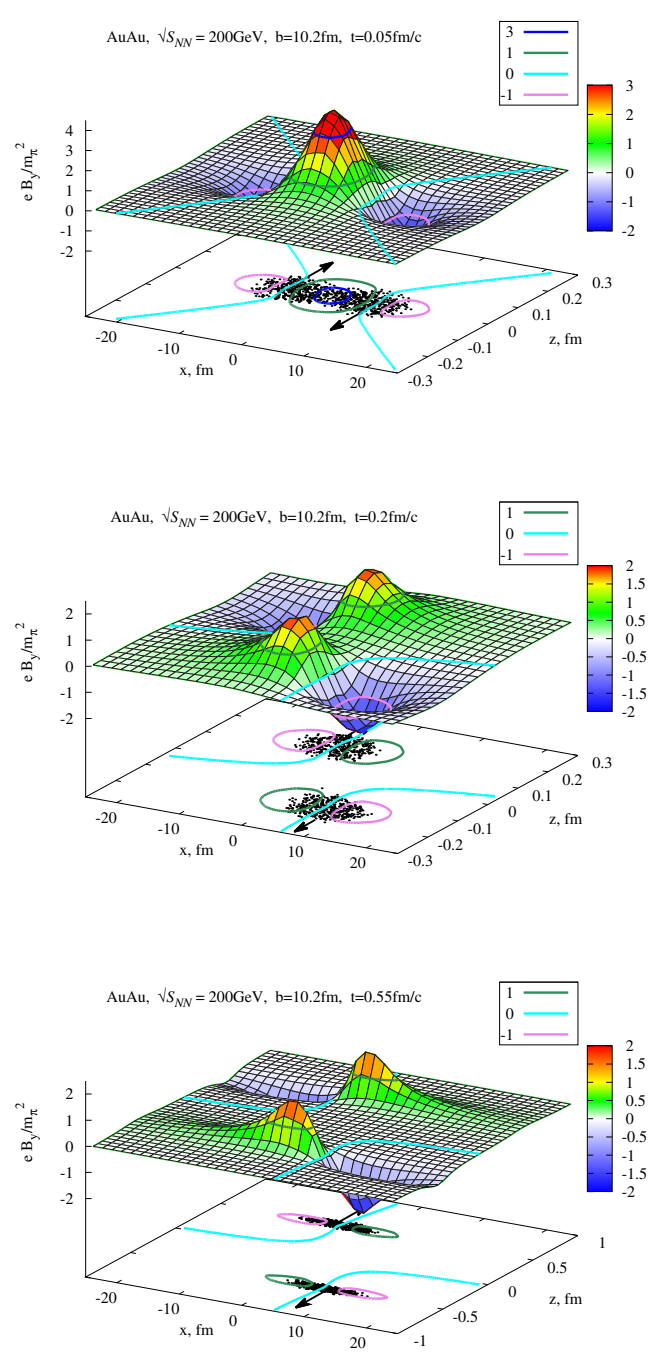

Fig. 6. Distribution of the magnetic field strength $e B_{y}$ in the $y=0$ plane at $t=0.05$ (upper panel), 0.2 (in the middle) and 0.55 (lower panel) $\mathrm{fm} / \mathrm{c}$ for Au+Au collisions at $\sqrt{s_{N N}}=200$ and $b=10.2$ $\mathrm{fm}$. The collision geometry is projected on $x-z$ plane by points corresponding to a particular spectator position. Curves (and their projections) are levels of the constant $e B_{y}$.

In this case Eq. (18) leads to the above used retarded Liénard-Wiechert potentials generated by every moving charged particle and acting at the point $\mathbf{R}=\mathbf{r}-\mathbf{r}^{\prime}$ at the moment $t$ [11].

One should note that the off-shell HSD transport approach is based not on the Boltzmann-like transport equation (14) but rather on the off-shell Kadanoff-Baym equations [16] having similar general structure. The set of equations was solved in a quasiparticle approximation by using the Monte-Carlo parallel ensemble method. To find the magnetic field a space grid was used. In a lattice point of this grid the retarded vector potential is calculated by numerical differentiation. The field inside a cell is approximated by that at the nearest grid point. To avoid singulari- 
ties and self-interaction effects, particles within a given cell are excluded from procedure of the field calculation.
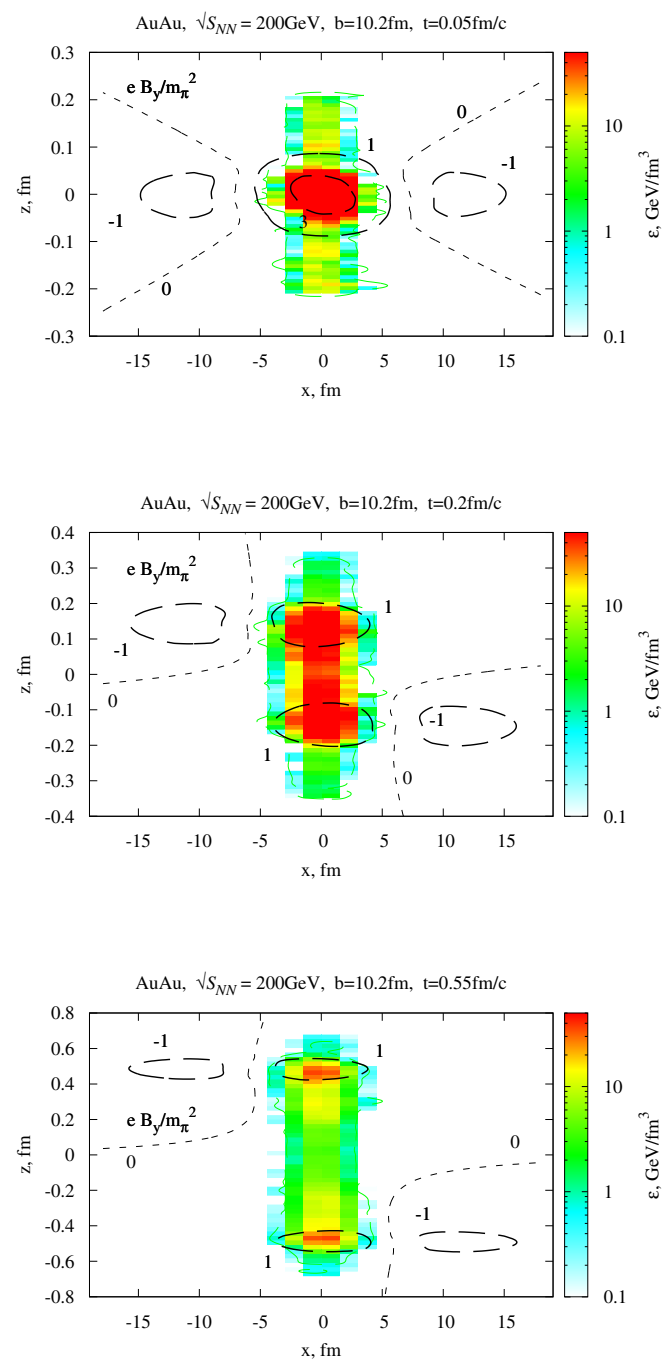

Fig. 7. The $x-z$ projection of the energy density $\varepsilon$ and magnetic field strength $e B_{y}$ (contour lines) in the $y=0$ plane for three cases presented in Fig.6.

An evolution snapshot of the magnetic field $B_{y}(x, y=$ $0, z, t)$ (in units of $m_{\pi}^{2}$ ) formed in $\mathrm{Au}+\mathrm{Au}(200 \mathrm{GeV})$ peripheral $(b=10.2 \mathrm{fm})$ collisions are given in Fig. 6 for three time moments $t=0.05,0.20$ and $0.55 \mathrm{fm} / \mathrm{c}$. The collisional geometry is presented by a set of points every of which corresponds to a spectator nucleons. The field is not homogeneous exhibiting a wide maximum over the transverse size of overlapping (participant) matter. Opposite rotation of the magnetic field along direction of two colliding nuclei results in corresponding two minima from outer sides of spectator matter remnants. At expansion these remnants are moving away from each other. It is of interest that for the fixed $x$ the width of $Z$ distributions of the magnetic field does not changed much. This type of scaling has been assumed in the phenomenological model of the CME considered above. The maximum in the magnetic field strongly correlates with that in the energy density of created particles as is clearly seen from Fig.7. Large local values of $B_{y}$ and $\varepsilon$ reached in these $\mathrm{Au}+\mathrm{Au}$ collisions provide necessary conditions for observation of signals of a possible parity violation.

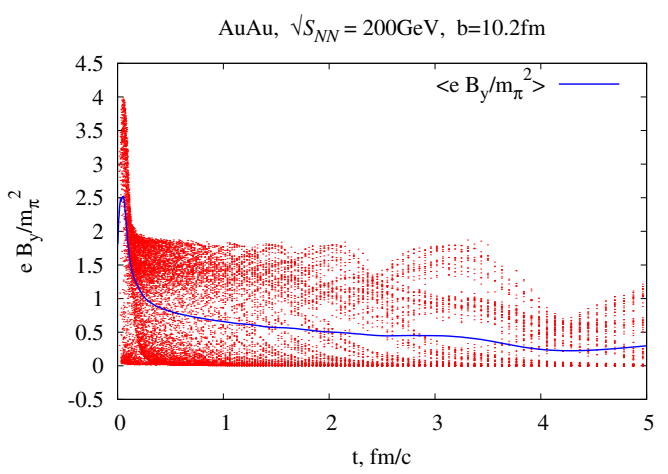

Fig. 8. The time evolution of the magnetic field $e B_{y}$ at the point where a pion is situated (shown by points). The curve corresponds to the field averaged over these points at every time moment.

A particular characteristic is shown in Fig.8. The magnetic field in the point where a real meson is situated is plotted as a function of time for every meson. The curve corresponds to the averaged field $\left\langle B_{y} / m_{\pi}^{2}>\right.$ at the given time moment. It is seen that averaged magnetic field sharply goes down till $B_{y}<m_{\pi}^{2}$ and then falls down quite slowly. The moment, when the expansion regime is changed, is of order of $2 R / \gamma_{c m} \sim 0.2 \mathrm{fm} / \mathrm{c}$.

\section{Discussion and conclusions}

Summarizing Sect.2, one should note that for heavy-ion collisions at $\sqrt{s_{N N}} \gtrsim 11 \mathrm{GeV}$ the magnetic field and energy density of deconfined matter reach very high values which seem to be high enough for manifestation of the Chiral Magnetic Effect. However, these are only necessary conditions. To estimate a possible CME a particular model is needed. For the average correlator our qualitative prediction $a^{2} \sim s_{N N}^{-1 / 8}$ has a rather small exponent but nevertheless it is too strong to describe observable energy behavior of the CME. This model energy dependence can be reconcile with experiment [6] by a detailed treatment of the lifetime taking into account both the time of being in a strong magnetic field and time evolution of the energy density in the QGP phase. For the chosen parameters we are able to describe data for $\mathrm{Au}+\mathrm{Au}$ collisions on charge separation at two available energies. We predict that the effect will be much smaller at the LHC energy and will sharply disappear near the top energy of SPS. Experiments at coming into operation Large Hadron Collider and that planned at RHIC by the Beam Energy Scan program [17] are of great interest since they will allow one to test the CME scenario 
and to infer the critical magnetic field $e B_{\text {crit }}$ governing by the spontaneous local $C \mathcal{P}$ violation.

All the results presented above are obtained under assumption that evolution of the electromagnetic field scales as that in the central point of the overlapping region. The fall of $B_{y}$ in time is caused be the fact that spectators are flying away. This assumption is getting worse for lower SPS energies what should be tested in more elaborated models.

The experimentally observed CME enhancement for $\mathrm{Cu}+\mathrm{Cu}$ collisions is related with the selection of different impact parameters for the same centrality at variation of a colliding system. However, it is not reduced to a purely geometrical effect.

The problem of parity violation in strong interactions and the related CME are actively debated now. In particularly, the important finding has been reported recently [18]. To distinguish between the CME and effects due to certain two-particle correlations it was proposed to analyze the strength of charge separation effect and its azimuthal correlation with the reaction plane. It turned out that the observable effect for like-sign pions is the sum of a large negative in-plane correlator and small positive out-of-plane one, i.e. the correlations for same charge pairs are mainly in-plane. For unlike pions both components are positive and approximately equal to each other. All that is puzzling since the CME is expected only for out-of-plane events. A full dynamical simulation is needed for heavy-ion collisions with inclusion of the magnetic field formation, its evolution and its impact on particle trajectories. First step in this direction has been made in Sec.3. Such approach allows one to calculate the CME background. It is of great interest that the discussed CME signal, the electric charge asymmetry with respect to the reaction plane, may originate not only from the spontaneous local CP violation but also be simulated by other possible effects. The search for alternative explanations and additional manifestations of local parity violation is underway [19-34]). It is important that the developed kinetic approach in principle allows one to simulate the Chiral Magnetic effect itself. This work is in progress.

\section{Acknowledgements}

Successful collaboration with D. Kharzeev, V. Skokov, E. Bratkovskaya, W. Cassing, V. Konchakovski and S. Voloshin is greately acknowledged. We are thankful to V. Koch, R. Lacey, I. Selyuzhenkov, O. Teryaev and J. Thomas for comments. V.T. is partially supported by the DFG grant WA 431 8-1 RUSS and the Heisenberg-Landau grant. V.V. acknowledges financial support within the "HIC for FAIR" center of the "LOEWE" program.

\section{References}

1. D. Kharzeev, Phys. Lett. B633 (2006) 260.

2. D. Kharzeev and A. Zhitnicky, Nucl. Phys. A797 (2007) 67.
3. D.E. Kharzeev, L.D. McLerran anf H.J. Warringa, Nucl. Phys. A803 (2008) 227.

4. K. Fukushima, D.E. Kharzeev, and H.J. Warringa, Phys. Rev. D78 074033 (2008) 074033.

5. D.E. Kharzeev, and H.J. Warringa, Phys. Rev. D80 (2009) 034028.

6. S. Voloshin for STAR Collaboration, talk at the Quark Matter 2009, arXiv:0907.2213; STAR Collaboration: B.I. Abelev, et al., arXiv:0909.1717; The STAR Collaboration: B.I. Abelev, et al., Phys. Rev. Lett. 103 (2009) 251601.

7. W. Ehehalt, W. Cassing, Nucl. Phys. A 602 (1996) 449; E. L. Bratkovskaya, W. Cassing, Nucl. Phys. A 619 (1997) 413; W. Cassing, E. L. Bratkovskaya, Phys. Rept. 308 (1999) 65.

8. S.A. Voloshin, Phys. Rev. C70 (2004) 057901.

9. D. Kharzeev and E. Levin, Phys. Lett. B523 (2001) 79.

10. D. Kharzeev and M. Nardi, Phys. Lett. B507 (2001) 121.

11. V. Skokov, A. Illarionov and V. Toneev, Int. J. Mod. Phys. A24 (2009) 5935.

12. S. A. Bass et al., Prog. Part. Nucl. Phys. 41 (1998) 255.

13. M. Bleicher et al., J. Phys. G25 (1999) 1859.

14. V.A. Okorokov, arXiv:0908.2522.

15. R. Vogt, Ultrarelatevistic heavy-ion collisions, (Elsevier, 2007) Ch.3.

16. W. Cassing and S. Juchem, Nucl. Phys. A665 (2000) 377; Nucl. Phys. A672 (2000) 417; Nucl. Phys. A677 (2000) 445.

17. G. Odyniec, http://omnis.if.ufrj.br/ sqm09/, see also http://drupal.star.bnl.gov/STAR/starnotes/public/sn0493.

18. A. Bzdak, V. Koch and J. Liao, Phys. Rev. C81 (2010) 031901, arXiv:1005.5380, arXiv:1008.4919.

19. P.V. Buividovich, M.N. Chernodub, E.V. Luschevskaya and M.I. Polikarpov, Phys.Rev. D81 (2010) 036007.

20. F. Wang, Phys. Rev. C81 (2010) 064902.

21. R. Millo and E. Shuryak, arXiv:0912.4894.

22. O.V. Rogachevsky, A.S. Sorin and O.V. Teryaev, arXiv:1006.1331.

23. W.-j. Fu, Y.-X. Liu, Y.-1. Wu, arXiv:1002.0418.

24. B. Keren-Zur and Y. Oz, arXiv:1002.0804.

25. M.N. Chernodub, arXiv:1002.1473.

26. S. Pratt, arXiv:1002.1758; S.Schlichting and S. Pratt, arXiv:1005.5341; arXiv:1009.4283

27. M. Asakawa, A. Majumder and B. Müller, Phys. Rev. C81 (2010) 064912.

28. S.-il Nam, Phys. Rev. D80 (2010) 045017; arXiv:0912.1933.

29. S. Voloshin, Phys. Rev. Lett. 105 (2010) 172301.

30. V. Orlovsky and V. Shevchenko, arXiv:1008.4977.

31. A.R. Zhitnitsky, arXiv:1008.3598.

32. B. Müller and A. Schäffer, arXiv:1009.1053.

33. S.W. Mages, M. Aicher and A. Schäffer, arXiv:1009.1495.

34. D.E. Kharzeev and D.T. Son, arXiv:1010.0038; D.E. Kharzeev, arXiv:1010.0943. 